\title{
The evolution of the adult head in Diptera
}

\author{
Katharina Schneeberg \\ Entomology Group, Institut für Spezielle Zoologie und \\ Evolutionsbiologie mit Phyletischem Museum, FSU Jena, 07743 Jena. \\ Email: katharina.schneeberg@gmx.de
}

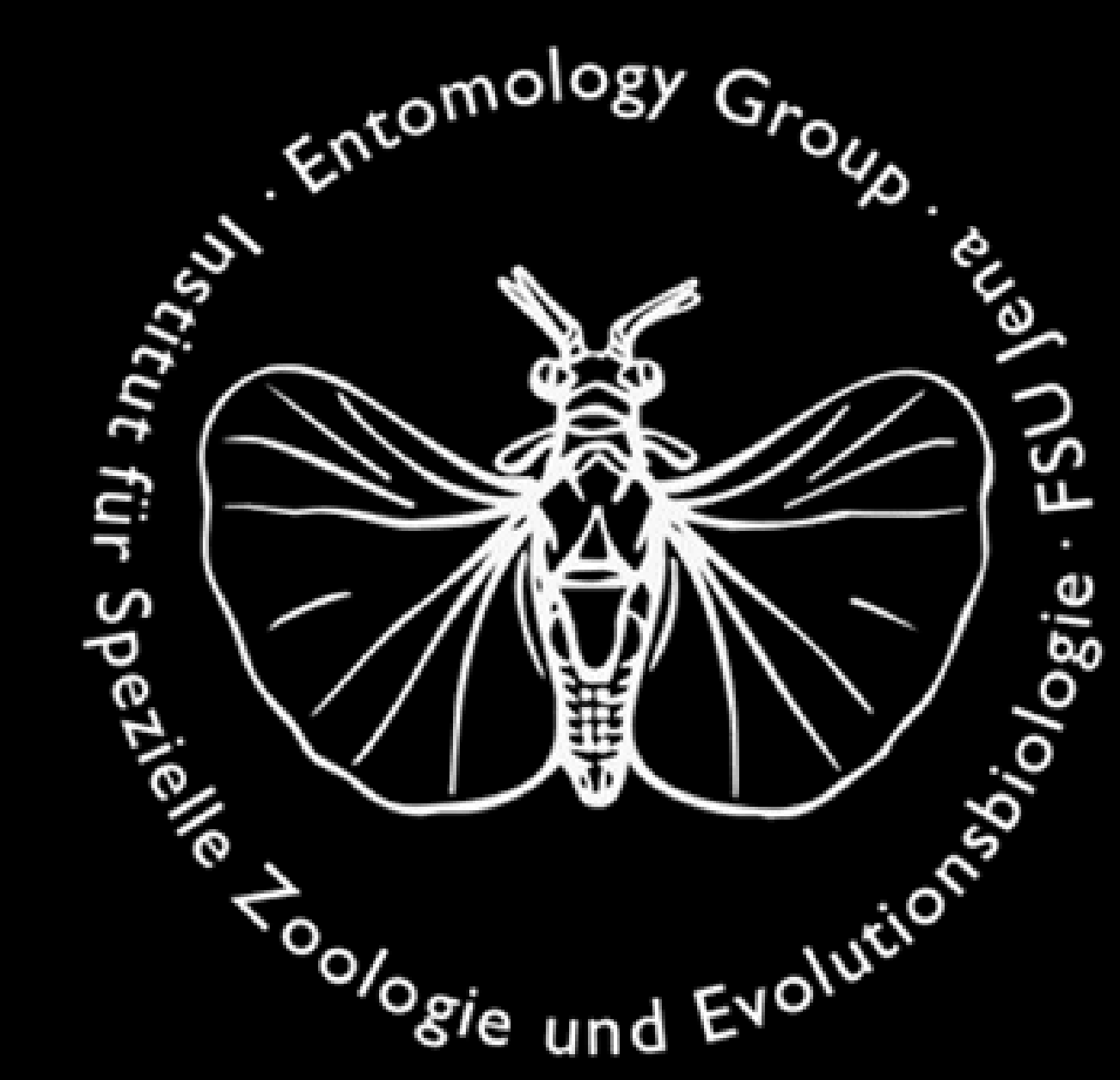

\section{Introduction}

Diptera is one of the species richest orders of holometabolous insects. They occur on all continents and are highly diverse in their ecology. Diptera are doubtlessly a monophyletic group and are traditionally subdivided into the two suborders "Nematocera" and Brachycera. The former group (named 'lower Diptera' in newer contributions) is a paraphyletic group, whereas Brachycera are monophyletic.

The head is an essential feature of adult insects, bearing a number of important sensory organs as well as containing the brain. In this study 36 taxa were investigated.
The taxon sampling includes at least one specimen from almost all families of 'lower Diptera', but also some brachyceran groups and outgroup taxa. The characters were mapped onto the phylogeny of Wiegmann et al. (2011) to develop an evolutionary scenario for the adult head structures.

\section{Results and discussion}

The head of adult Diptera is characterized by many reductions. Mandibles and their associated musculature are present in the groundplan of the order, but missing in most groups. The presence of mandibles is directly correlated with the feeding habit (see tree). They are present in bloodsucking species, like members of Culicidae, Ceratopogonidae or Tabanidae. In most dipteran groups thetentoriumisastraighthollowtube, connecting anterior and posterior tentorial pits. In some specimens the tentorium is completely reduced (Nymphomyiidae or Tipulidae). However, in the dipteran groundplan anterior, posterior and dorsal arms are well developed (present in Simuliidae and Tabanidae), but a typical corpotentorium is missing in all groups. The tendency to shift the origin of the antennal muscles from the tentorium to the head capsule is correlated with the far reduction of the tentorium, but without a recognizable phylogenetic pattern. The potentially basal families of Diptera, Deuterophlebiidae and Nymphomyiidae, are extremely specialized in their morphology and life cycle. Thereby the interpretation of the features appears difficult.

\section{Reference}

Wiegmann, BM et al. 2011. Episodic radiations in the fly tree of life. PNAS 108: 5690-5695.

\section{Acknowledgements}

I thank my supervisor Rolf Beutel (FSU Jena) for his support. I also thank Greg Courtney (lowa State University) and Eric Anton (Jena), who provide valuable material. Suggestions and comments made by Frank Friedrich (Uni Hamburg) and Maximilian Fraulob (FSU Jena) have helped to improve the layout. This study was funded by the DFG, this is greatfully acknowledged.
Mecoptera

Siphonaptera

Deuterophlebiidae

Nymphomyiidae

Trichoceridae

Pediciidae

Limoniidae

Cylindrotomidae

Tipulidae

- Ptychopteridae

Blephariceridae

Tanyderidae

Psychodidae

Ceratopogonidae

Thaumaleida

Simuliidae

Dixidae

Culicidae

Chaoboridae

Corethrellidae

Anisopodidae

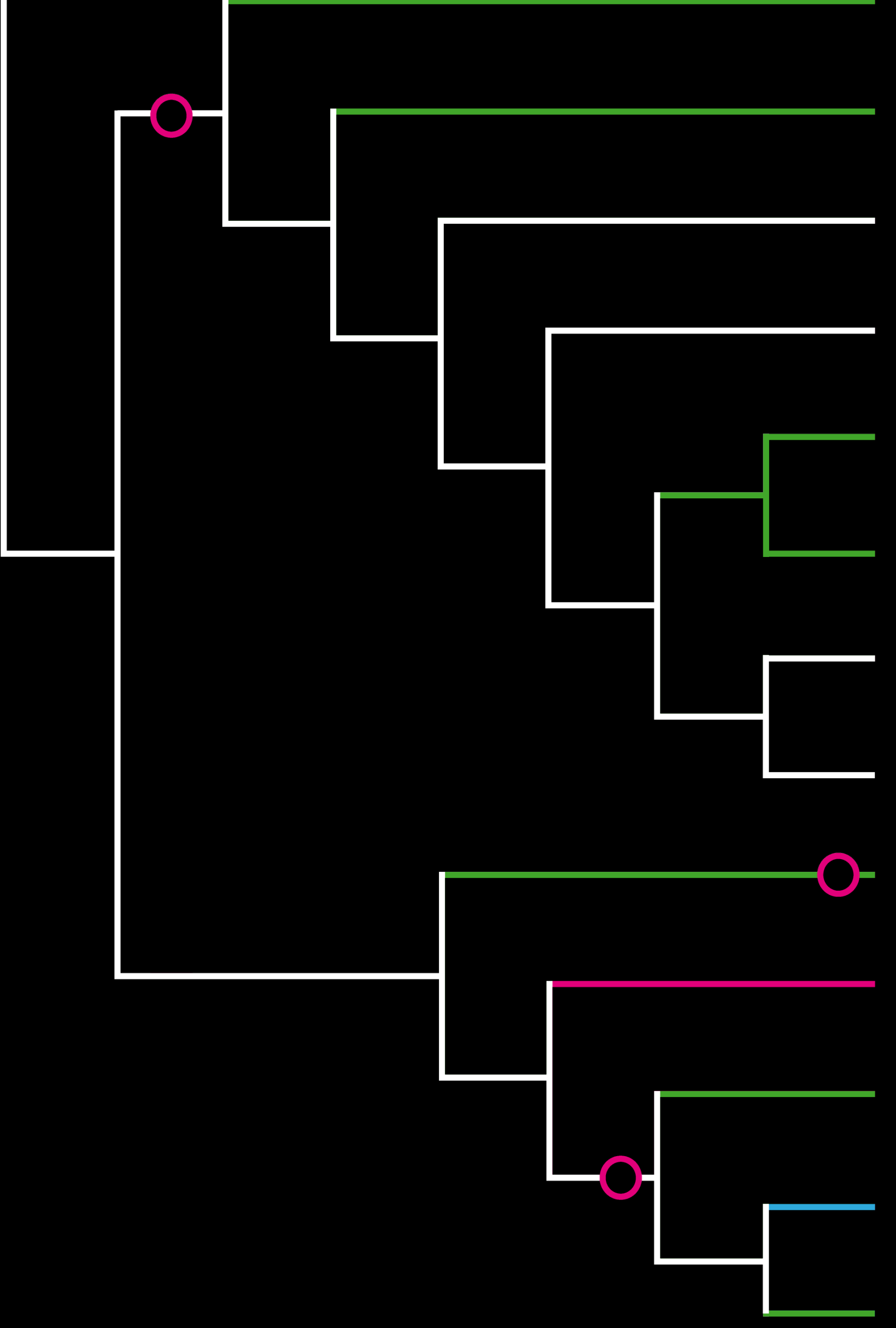

Scatopsidae

Axymyiidae

Bibionidae

Keroplatidae

Mycetophilidae

Sciaridae

Cecidomyiidae

Syrphidae

Tabanidae

Stratiomyiidae

Asilidae

Bombyliidae
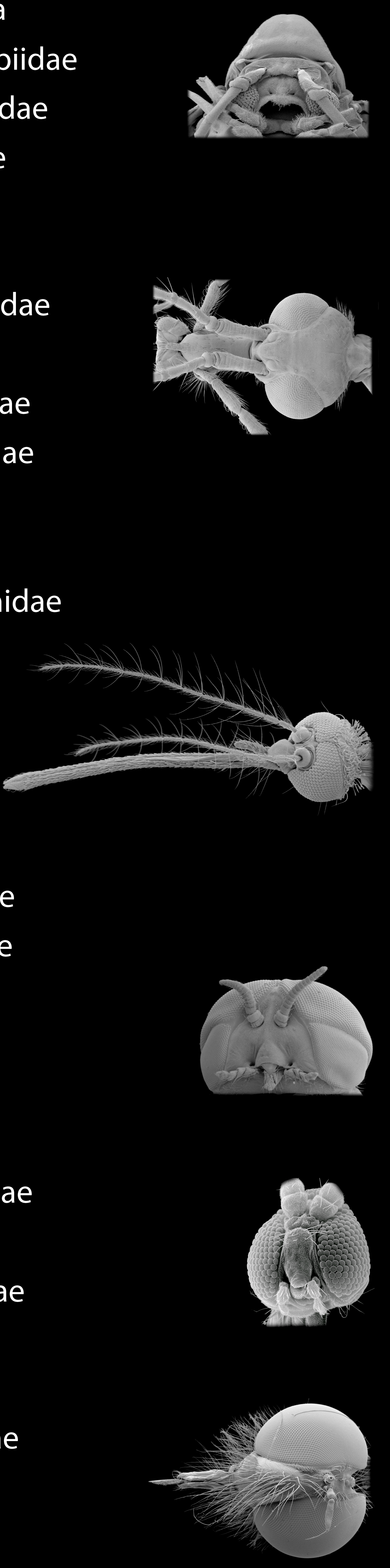

Characters mapped on Wiegmann et al. 2011 (modified). Branch color shows feeding habit: Blue - predatory, pink bloodsucking, brown - take up no food, green - plant jouces, white - unknown. The pink circles shows the presence of mandibles: - mandible present, $\bigcirc$ - mandible absent. SEM-images from top to bottom: Deuterophlebia (Deuterophlebiidae), Tipula (Tipulidae), Culex (Culicidae), Axymyia (Axymyiidae), Mayetiola (Cecidomyiidae), Silpnogaster (Asilidae). 